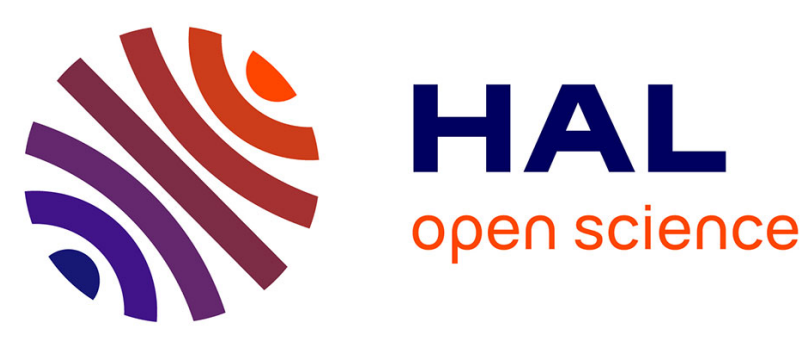

\title{
Posterior lobules of the cerebellum and information processing speed at various stages of multiple sclerosis
} Amandine Moroso, Aurélie Ruet, Delphine Lamargue-Hamel, Fanny Munsch,

Mathilde Deloire, Pierrick Coupé, Jean-Christophe Ouallet, Vincent Planche, Nicolas Moscufo, Dominik S Meier, et al.

\section{To cite this version:}

Amandine Moroso, Aurélie Ruet, Delphine Lamargue-Hamel, Fanny Munsch, Mathilde Deloire, et al.. Posterior lobules of the cerebellum and information processing speed at various stages of multiple sclerosis. Journal of Neurology, Neurosurgery and Psychiatry, 2016, 10.1136/jnnp-2016-313867 . hal01398727

\section{HAL Id: hal-01398727 \\ https://hal.science/hal-01398727}

Submitted on 17 Nov 2016

HAL is a multi-disciplinary open access archive for the deposit and dissemination of scientific research documents, whether they are published or not. The documents may come from teaching and research institutions in France or abroad, or from public or private research centers.
L'archive ouverte pluridisciplinaire HAL, est destinée au dépôt et à la diffusion de documents scientifiques de niveau recherche, publiés ou non, émanant des établissements d'enseignement et de recherche français ou étrangers, des laboratoires publics ou privés. 


\section{Posterior lobules of the cerebellum and information processing speed at various $\underline{\text { stages of multiple sclerosis }}$}

\section{AUTHORS}

Amandine Moroso, MSc, ${ }^{1,23}$ Aurélie Ruet, MD, PhD, ${ }^{1,2,3}$ Delphine Lamargue-Hamel, $\mathrm{PhD}{ }^{1,3}$ Fanny Munsch, $\mathrm{PhD}^{1,3}$ Mathilde Deloire, $\mathrm{PhD},{ }^{2}$ Pierrick Coupé, $\mathrm{PhD},{ }^{1,4}$ Jean-Christophe Ouallet, MD, PhD, ${ }^{2}$ Vincent Planche, MSc, ${ }^{1,3}$ Nicolas Moscufo, PhD, ${ }^{5}$ Dominik S. Meier, PhD, ${ }^{5}$ Thomas Tourdias MD, PhD,${ }^{1,2,3}$ Charles R.G.

Guttmann, MD ${ }^{5}$ Vincent Dousset, MD, PhD, ${ }^{1,2,3}$, Bruno Brochet, MD. ${ }^{1,2,3}$

\section{AUTHORS' AFFILIATIONS}

1 Univ. Bordeaux, F-33076 Bordeaux, France,

2 CHU de Bordeaux, INSERM-CHU CIC-P 0005, \& Services de Neurologie et Neuroradiologie, F-33076

Bordeaux, France,

3 Neurocentre Magendie, INSERM U1215 F-33077 Bordeaux, France,

4 LaBRI, UMR 5800, PICTURA, F-33400 Talence, France,

5 Center for Neurological Imaging, Department of Radiology, Brigham and Women's Hospital, Harvard Medical School, Boston, Massachusetts, USA.

\section{CORREPONDING AUTHOR}

Bruno Brochet, Department of Neurology, CHU de Bordeaux, 33076 Bordeaux cedex, France. Tel :

$+33556795521$

Email: bruno.brochet@chu-bordeaux.fr

\section{AUTHORS' CONTRIBUTION}

Drafting the manuscript: AM and BB. Revised the manuscript for important intellectual content: all authors. Study concept and design: AM, AR, DLH, MD and BB. Analysis and interpretation of the data: AM, AR, DLH, FM, MD, PC, NM, DSM, TT, CRGG and BB. Acquisition of the data: AR, MD, DLH and BB. Statistical analysis: AM, MD. Study supervision and coordination: BB, AR, TT.

\section{WORDS COUNT 3187}

ABSTRACT 247 



\section{ABSTRACT:}

Background: Cerebellar damage has been implicated in information processing speed (IPS) impairment associated with multiple sclerosis (MS) that might result from functional disconnection in frontocerebellar loop. Structural alterations in individual posterior lobules, in which cognitive functioning seems preponderant, are still unknown. Our aim was to investigate the impact of grey matter (GM) volume alterations in lobules VI to VIIIb on IPS in persons with clinically isolated syndrome (PwCIS), MS (PwMS) and healthy subjects (HS).

Methods: Sixty-nine patients (37 PwCIS, 32 PwMS) and 36 HS underwent 3T magnetic resonance imaging including 3D T1 weighted MR images. Cerebellum lobules were segmented using SUIT 3.0 to estimate their normalized GM volume. Neuropsychological testing was performed to assess IPS and main cognitive functions.

Results: Normalized GM volumes were significantly different between PwMS and HS for the right $(\mathrm{p}<0.001)$ and left lobule VI $(\mathrm{p}<0.01)$, left Crus I, right VIIb and entire cerebellum $(\mathrm{p}<0.05$ for each comparison) and between PwMS and PwCIS for all lobules in subregions VI and left Crus I $(p<0.05)$. IPS, attention, and working memory were impaired in PwMS compared to PwCIS. In the whole population of patients (PwMS and PwCIS) GM loss in Vermis VI $\left(\mathrm{R}^{2}=0.36 ; \mathrm{p}<0.05\right.$ when considering age and $\mathrm{T} 2$ lesion volume as covariates $)$ were associated with IPS impairment.

Conclusions: GM volume decrease in posterior lobules (especially Vermis VI) were associated with reduced IPS. Our results suggest a significant impact of posterior lobules pathology in cortico-cerebellar loop disruption resulting in automation and cognitive optimization lack in MS. 
The mechanisms involved in cognitive impairment (CI) of persons with multiple sclerosis (PwMS) are not fully understood.(1) CI affects several cognitive domains, including episodic memory, attention, working memory and executive functions.(1)(1) Slowing of information processing speed (IPS) is the main cognitive dysfunction observed in MS even at early stages.(2) Magnetic resonance imaging (MRI) studies suggested that it may be related to distributed demyelinating and neurodegenerative alterations of brain networks. $(3,4)$ The cerebellum could be part of these networks and the involvement of cerebellar dysfunction in IPS impairment has been suggested.(5) A role for the cerebellum in cognition in healthy subjects (HS) has been suggested by anatomical, clinical and imaging studies.(6-10) The cortico-cerebellar loop connects posterior cerebellar hemispheres and dentate nuclei to prefrontal, superior temporal and lateral parietal cortices, via the thalamus and the pons.(6) A precise cerebellar functional map has emerged from different functional MRI (fMRI) studies leading to the idea of dedicated cognitive lobules in the most posterior parts of the cerebellum.(11) Cerebellar damage is common in MS and extensive demyelination has been described in the cerebellar cortex on postmortem samples.(12) Some studies showed an association between cognitive impairment, including IPS, and cerebellar symptoms in PwMS.(5,13) An association between cerebellar atrophy, especially GM global atrophy, and IPS and working memory impairment have been shown in MS.(14-18) Several fMRI studies in HS and in PwMS have shown that greater cerebellar activation correlates with faster cognitive performances in HS but not in PwMS and showed that the functional link between the cerebellum and frontal areas was impaired in MS patients.(19-21) The decrease in cerebellar activation in PwMS has been interpreted as a failure of the cerebellum in facilitating rapid cognitive performances and may play a role in diminished IPS in MS.

Since the posterior cerebellar lobules that have been specifically implicated in cognition and are anatomically connected to the frontal areas implicated in cognitive networks, we hypothesized that posterior cerebellar GM damage could be responsible for cortico-cerebellar disconnection and thus lack of cognitive optimization and automation resulting in IPS impairment. In pediatric MS the global volume of posterior lobules of the cerebellum have been correlated with cognition including IPS.(18) Our study aimed to investigate the impact of GM volume alterations in individual lobules VI to VIIIb on cognitive outcome, especially IPS, in persons with clinically isolated syndrome (PwCIS), PwMS and HS.

\section{METHODS}

\section{Subjects}

Sixty nine patients (PwCIS or PwMS) and 69 HS, matched for age, sex and educational level were 
recruited between June 2010 and December 2014 at the Bordeaux University Hospital Center, France. All patients, as well as 36 out of 69 HS underwent an MRI scan and all study participants were evaluated with cognitive testing.

All PwCIS ( $\mathrm{n}=37$ ) were included within 6 months after their first neurological episode and presented at least two asymptomatic cerebral lesions on Fast Fluid-Attenuated Inversion-Recovery (FLAIR) images. For PwMS ( $\mathrm{n}=32$ ) the inclusion criteria were as follows: MS diagnosis according to McDonald's criteria,(22) disease duration $>6$ months and $\leq 15$ years, and mild cognitive impairment defined as two scores beyond one standard deviation (SD) among a large neuropsychological battery. MS patients were treated according to current standards of clinical care.

Exclusion criteria were: age under 18 or over 55 years, history of other neurological or psychiatric disorder, inability to perform computerized tasks or MRI, MS attack in the two months preceding the screening, corticosteroid pulse therapy within two months preceding screening, severe cognitive deficits (Mini-Mental State Examination <27), depression (Beck Depression Inventory score (BDI) >27).

Clinical assessment, the French version of Expanded Disability Status Scale (EDSS) and the Multiple Sclerosis Severity Score (MSSS)(23) were determined by expert neurologists.

\section{Standard protocol, approvals, registration, and patient consents}

Each subject gave written informed consent. Patients were included from two different studies (REACTIV, ClinicalTrials.gov Identifier: NCT01207856, study concerning cognitively impaired PwMS, and SCI-COG, ClinicalTrials.gov Identifier: NCT01865357, analyzing cognitive impairment in PwCIS). Both studies were approved by the local ethics committee.

\section{Neuropsychological assessment}

Each cognitive domain was evaluated with the following tests (CIS and paired HS: *; MS and paired HS: ${ }^{* *}$ ). Because PwCIS and PwMS were included in two different studies, a few tests, only for working memory and verbal fluency, differed between the two samples, and z scores for cognitive domains were calculated by comparisons with scores obtained in the control group. IPS tests were the same for all participants. Tests used for neuropsychological assessment are described in a previous work.(24)

1. Attention: Test of Attentional Performance (TAP) ${ }^{* * * *}$ consisting of subtests for accurate answers of visual scanning, and visual and auditory divided attention. For divided attention, the number of 
accurate answers and reaction time ratios of the double task (auditory and visual divided attention) to the simple task (auditory or visual divided attention) were considered,

2. Working Memory: Numerical span test (forward ${ }^{* / * *}$ and backward ${ }^{* / * *}$ ) and Paced-Auditory Serial Addition Test-3 seconds (PASAT) ${ }^{*}$ or working memory subtest of the TAP**,

3. Executive functions: Stroop test ${ }^{* * *}$ (using the difference between the denomination part and the inhibition task scores) and Word List Generation test (verbal fluency assessment) ${ }^{*}$ or semantic verbal fluency (using animal category) ${ }^{* *}$,

\section{IPS: Symbol Digit Modalities Test (SDMT)*/**}

Depression and anxiety levels were assessed using the BDI, and State-Trait Anxiety Inventory for adults, subtest State (STAI-S), respectively.

\section{MRI acquisition}

MRI scans were performed on a 3T Achieva TX system (Philips Healthcare, Best, The Netherlands) with an 8-channel phased array head coil. A morphological protocol consisted of: 3D T1 weighted MR images acquired using magnetization prepared rapid gradient echo $(\mathrm{MPRAGE})$ imaging $(\mathrm{TR}=8.2 \mathrm{~ms}, \mathrm{TE}=3.5 \mathrm{~ms}, \mathrm{TI}=$ $982 \mathrm{~ms}, \alpha=7^{\circ}, \mathrm{FOV}=256 \mathrm{~mm}$, voxel size $=1 \mathrm{~mm}^{3}, 180$ slices $)$ and $2 \mathrm{D}$ multi-slice FLAIR images $(\mathrm{TR}=11000 \mathrm{~ms}$, $\mathrm{TE}=140 \mathrm{~ms}, \mathrm{TI}=2800 \mathrm{~ms}, \mathrm{FOV}=230 \mathrm{~mm}, 45$ axial slices, $3 \mathrm{~mm}$ thick$).$

\section{Post processing and Image Analysis}

\section{Volumetric analysis}

Total brain, GM and white matter (WM) volumes, normalized for skull size in the Montreal Neurological Institute (MNI) space, were estimated from 3DT1 MPRAGE images using SIENAX(25) (part of FSL 5.0, with brain extraction tool (BET) options: "-B -f 0.3"), taking into account FLAIR lesion masks for patients. A custom image processing pipeline was applied to automatically segment the WM lesions. A corrected Freesurfer parcellation was used on the T1-weighted MRI to obtain spatial priors for WM, GM and cerebrospinal fluid.(26) A 2-channel Expectation-Maximization algorithm produced the final segmentation of WM lesions as outliers of the WM intensity distributions.(27) The output of the pipeline is a WM lesion probability map from which an expert user derived the final binary map by a lesion probability thresholding step using 3D Slicer v4.0.

For cerebellar volumes estimations, cerebellar GM and WM probability maps were obtained as well, using BET and FAST (FSL 5.0). SUIT toolbox 3.0 (SPM 8)(28) was used for cerebellar lobule segmentation. 
The software allows standardizing lobule size to drive a reliable segmentation independent of inter-individual variability, and set back the images in each patients' native space in order to calculate lobular volumes. Steps are described below (Figure 1):

1. The cerebellum was identified on brain on 3D-T1 MPRAGE images,

2. Cropped T1 weighted images underwent a non-linear registration to the SUIT template,

3. The same non-linear registration was performed on WM/GM probability maps described above, using $7^{\circ} \mathrm{B}$-spline interpolation in order for lobules of all subjects to be in a normalized coordinate space,

4. A reverse non-linear registration was performed for probability maps, a cerebellar lobule atlas (CerebellumSUIT) and a whole cerebellum atlas (Buckner) into each patient's native space in order to obtain total cerebellar and lobular volumes, as well as the volumes for their WM and GM compartments for each subject.

Using FSL 5.0, GM and WM probability maps were thresholded in order to include the whole cerebellar volume without any overlap between GM and WM and to create binarized label maps. Atlases previously registered in patients' native space were also binarized in order to differentially label each lobule. The total, GM and WM volumes were then calculated for the whole cerebellum, as well as for each lobule using MATLAB 2011b. All volumes were normalized for skull size, using the SIENAX scaling factor.

Lobules VI, Crus I, Crus II, VIIb, VIIIa and b were studied because of their known involvement in cognition. Vermis Crus I was excluded from analyses as some subjects were lacking reliable segmentation maps due to this structure's small size.

\section{Statistical analysis}

All data were analyzed with R package 'stats' (version 3.1.3). Normal distribution was tested for all variables with the Shapiro-Wilk test.

Sex and educational level were compared using the $\mathrm{Chi}^{2}$ test. Quantitative clinical and imaging data were compared between PwCIS, PwMS and HS with ANOVA or Kruskal-Wallis tests depending on their distributions. For post hoc analyses, Tuckey or Nemenyi tests were used to compare two subgroups when ANOVA or Kruskal-Wallis tests respectively showed significant results.

Z scores were calculated according to the formula: (patient's raw score - HS mean score / HS standard deviation (SD)) using our population of $69 \mathrm{HS}$. Cognitive domain impairment was defined by a z score below -1.5 in the given domain. Z score comparisons between PwMS and PwCIS were obtained with paired t-test (or Mann-Whitney). A 
significance threshold of 0.05 was applied.

According to the variables' distribution, Spearman or Pearson correlations between imaging and cognitive outcome in all patients (CIS and MS), and PwCIS and PwMS independently, were used and then adjusted for multiple comparisons using a Bonferroni-adjusted significance threshold of $\mathrm{p}<0.002$.

Linear regression analysis was used to predict cognitive outcome, including three hierarchical blocks 1) Clinical data (age and MSSS), 2) Cerebellar volumes (lobule GM volumes and whole infratentorial T2-lesion volume), 3) Cerebral volumes (cerebral WM, cerebral GM and cerebral T2-lesion volume). Dependent variable and residual normal distributions were checked using the Shapiro test and histogram analyses. Independent variables were entered in the model only if the related $p$ value was below 0.10 in univariate analyses.

\section{RESULTS}

\section{Demographics, Clinical data and Cognitive assessment}

We included 37 PwCIS, 32 PwMS and 69 HS. There were no significant differences in sex, median age and educational level between groups, either when considering the whole HS group or only the HS subgroup that underwent MRI. Table 1 describes population demographics and clinical characteristics.

No PwCIS and $6.25 \%$ of PwMS were impaired on attention compared with HS. Working memory impairment occurred in $5.41 \%$ of PwCIS and $25 \%$ of PwMS, executive functions impairment in $8.11 \%$ of PwCIS and $15.63 \%$ of PwMS, IPS slowness occurred in $13.51 \%$ of PwCIS and $71.77 \%$ of PwMS. Attention, working memory and IPS mean z scores were significantly decreased in PwMS versus PwCIS. No differences were detected for executive functions.

No correlation was found between cognitive assessment and anxiety, depression scales or MSSS.

\section{Volumetric analysis}

Table 2 shows that normalized cerebral and cerebellar volumes were significantly different between groups. The PwMS group developed significant GM atrophy in whole cerebellum, as well as the cerebrum. Interestingly, within the cerebellum, atrophy was observed in several sub-structures i.e. lobules VI, left Crus I, and the right VIIb. The PwCIS group had preserved GM volumes compared with HS.

\section{Correlations between Cognitive outcomes and Imaging data}

Results of univariate correlations between imaging data and cognitive outcomes and multiple linear 
regressions are presented in Table 3. In the whole group of patients (MS and CIS, n=69), normalized GM matter volumes in the whole cerebellum, vermis VI, and bilateral Crus II were significantly associated with IPS z scores, and a trend was also observed for all posterior lobules. No significant correlation was found between other cognitive domains and GM volume in posterior lobules.

According to multivariate analysis, the Right VIIb volume $\left(\mathrm{R}^{2}=0.09 ; \mathrm{F}=7.98 ; \mathrm{p}<0.01\right)$ was associated with working memory, while age, vermis VI GM volume and cerebral T2-lesion volume $\left(\mathrm{R}^{2}=0.36 ; \mathrm{F}=13.30\right.$; $\mathrm{p}<0.001)$ were associated with IPS z scores. Infratentorial T2-lesion volume was correlated with executive functions. No model was significant to predict attentional outcome.

\section{DISCUSSION}

We found evidence that cerebellar atrophy in specific posterior cerebellar lobules had an impact on cognitive outcome in MS, specifically on IPS, even though global cerebellar atrophy did not show a significant independent correlation with cognition. Indeed, GM loss in vermis VI was associated with IPS impairment. This result supports our hypothesis that a lack of optimization and automation role of the posterior substructures of the cerebellum, and especially vermis VI, may induce IPS slowness. The correlation observed between right VIIb GM volume decrease and working memory impairment was probably not clinically relevant due to the very low value of the R2 coefficient, but could reflect an effect of IPS impairment on working memory which has been previously discussed by others.(29)

Information processing speed is strongly related to other cognitive domains and particularly executive functioning. Many studies have related the impact of cerebellar posterior lesions on executive functioning such as strokes, tumors, postinfectious cerebellitis, neurogenetic cerebellar syndromes, or cerebellar congenital malformations.(10,30) In their first description of the cerebellar cognitive affective syndrome (CCAS), $\underline{\text { Schmahmann et al. have found that among twenty patients suffering from cerebellar alterations, eighteen had }}$ executive impairment. In this study, patients free from such processing troubles had anterior and no posterior cerebellar damages, leading to motor rather than cognitive disability.(30)

However, CCAS not only consist in executive dysfunctions but also include visual spatial, and $\underline{\text { linguistic impairments, and affective dysregulation, whereas remote episode and semantic memory relatively }}$ preserved.(30,31) Studies in MS patients corroborate this description. Weier et al. demonstrated that cerebellar $\underline{\text { T1 lesion volume correlated with working memory (PASAT).(15) Patients with cerebellar lesions also displayed }}$ attentional, verbal fluency and spatial memory deficits compared to lesion free patients and healthy subjects.(32) 
Cerebellar WM matter lesions contribute to cognitive disability but did not seem to be the leading cause of cognitive impairment in MS. In their papers, Van de Pavert et al. and Morgen et al. correlated working memory (PASAT)(14,17), executive functions and attention performances(17) to GM atrophy in the whole cerebellum. In our study the whole cerebellar volume correlated with cognitive scores in univariate analyses but not in the multivariate models, suggesting that the association was mainly driven by the volume of the posterior lobules. We observed preponderant GM atrophy in PwMS but not in PwCIS, in agreement with previous studies, $(33,34)$ whereas another study detected atrophy at this stage only when disease duration was not taken into account.(35) We observed a non-significant trend of an increase of GM cerebellar volume in PwCIS in agreement with a longitudinal study showing a transient paradoxical increase in cerebellar GM volume in PwCIS possibly due to inflammation, preceding atrophy.(36) In RRMS a majority of studies tend to show cerebellar GM atrophy.(15,16,33,34) In MS patients with cerebellar symptoms a specific pattern of fronto-temporal cortical atrophy, independent of cerebellar lesions has been described highlighting cortico-cerebellar disconnection in MS.(37)

As mentioned above, this disconnection seem to occur between associative cerebral cortices and posterior cerebellar lobules specifically.(8) In HS, although all posterior lobules are engaged, some preferential contribution of specific lobules in cognitive domains have been observed, such as the left supero-posterior cerebellum for attention,(7) vermis VI and Crus I for verbal working memory and lobule VI, Crus I/II and VIIb for executive functions. $(9,8)$ For MS patients no such anatomical associations have previously been investigated. Our results highlighted the relationship between specific posterior cerebellar GM alterations and IPS rather than a specific cognitive domain. It is, however possible that at later stages of the disease cerebellar pathology contributes more significantly to CI in other domains. We found that GM loss in vermis VI was more specifically associated with IPS impairment. Vermis VI involvement has been documented in implicit memory and sequence learning reaction time, supporting its role in IPS.(38) Moreover, lobule VIIb volume, associated with working memory in our results, has been previously linked to executive functions and language, notably concerning the right side which is connected to the left cerebral associative cortices. $(8,9)$ Finally, Crus II was associated with IPS in univariate analysis but not in multivariate models. Crus II is less involved in cognitive processing in literature, consistent with our findings.(8)

Associative cerebellar processes are involved in selecting, optimizing and automating cognitive processes rather than integrating cognitive tasks, which is the prefrontal cortex' main role. In HS, fMRI studies suggested that speeder cognitive performances are associated with greater activation in the cerebellum and 
disclosed functional connectivity between the cerebellum and the dorso-lateral prefrontal areas during cognitive tasks.(39) The cerebellum may assume the most automatized parts of attentional requests which are needed to perform cognitive tasks in order to spare cortical regions. Cerebellar processes leading to cognitive automation and optimization are mostly unknown. However, analogous to motor skills, theories have emerged considering a transition from "controlled" to "automatic" cognitive functioning throughout learning processing. Hence, due to cerebellar modulation, tasks that require high cognitive load and complex attention become stereotyped, independent from distraction and from high-level cerebral associative oversight. Posterior cerebellar lobules seem to integrate internal representations with external stimuli in order to generate the proper response, progressing efficiently according to the context. On a physiological point of view, the cerebellar cortex consists of a homogenous histological organization independent from sensori-motor or cognitive functioning. Cerebellar inputs are then responsible for functional specialization. Concerning cognitive adaptation, theoretical models suggest that this phenomenon implies synaptic plasticity between Purkinje cells and prefrontal and parietal projections. Once afferent information from prefrontal and parietal cortices is selected and compared to internal representation, synaptic reinforcement permits to strengthen the most adapted comportment. Associative cerebral cortices are then disengaged from the automatic and harmonious generated response, resulting in faster reaction time and possibility to perform new high competence behavior.(40) According to Schmahmann et al. dysfunctions of this homeostatic control may lead to "dysmetria of thoughts". The mechanisms by which cerebellar damage could be associated with IPS impairment have been suggested by several fMRI studies.(1921) In early-RRMS, when patients showed normal performances in the easiest levels of the tasks and significantly longer reaction times (slow IPS) during the most demanding cognitive conditions, shorter reaction times were not associated with higher cerebellar activation but with an increase in medial prefrontal activation instead and the functional connectivity analysis showed a functional link between the dorso-lateral prefrontal cortex and medial prefrontal regions but not with the cerebellum.(19) These results suggested that MS patients are unable to activate the typical cerebello-frontal network associated with the fastest responses in the task and that they activate a substitute compensatory network involving the medial prefrontal cortex.

Our study is not without limitations. Volumetric analysis is challenging because of inter-individual variability. Moreover, thresholding at 0.5 was used to discriminate between GM and WM with a possible overestimation of cerebellar WM. However, we checked every segmentation output and, when necessary, manually corrected mismatches in GM and WM in the 3D-T1 sequences and these limitations did not have an 
impact on our results.

\section{CONCLUSION}

This study reinforces and extends the suggestion made by previous works of an important role for cerebellar damage in cognitive impairment at different stages of MS. For the first time, alterations in specific posterior lobules (especially Vermis VI) were associated with IPS impairment, suggesting an important role for alterations of these structures in this impairment due to a lack of cognitive automation and optimization through anatomical disconnection in the cortico-cerebellar loop.

\section{ACKNOWLEDGMENTS}

A. Moroso received research grant from the Fondation pour la Recherche Médicale (DEA20140630564), This study was supported by ANR-10-LABX-57 Translational Research and Advanced Imaging Laboratory (TRAIL), laboratory of excellence. SCICOG study was also supported by a grant from Teva and REACTIV study by a grant from Merck-Serono. CRG Guttmann was supported in part by a grant from the National Multiple Sclerosis Society (grant identifier RG-1501-03141) and by a visiting professorship grant from the Excellence Initiative (IdEx) of the University of Bordeaux. The sponsors did not participate in any aspect of the design or performance of the study, including data collection, management, analysis, and interpretation or the preparation, review, and approval of the manuscript.

\section{CONFLITS OF INTEREST AND DISCLOSURES}

Pr Brochet, Dr Ruet and Dr Ouallet or their institution received research grants and/or consulting fees from Biogen-Idec, Bayer-Healthcare, Novartis, Genzyme, Roche, Medday, Merck-Serono and Teva,

A. Moroso, D. Hamel, F. Munsch, M. Deloire, P. Coupé, , N. Moscufo, D. Meier, T. Tourdias, V. Dousset have nothing to disclose

C. Guttmann received a research grant from Sanofi.

V. Planche received travel expenses from ARSEP Fondation, Biogen, Teva-Lundbeck and Merk-Serono.

\section{REFERENCES}

1. Chiaravalloti ND, DeLuca J. Cognitive impairment in multiple sclerosis. Lancet Neurol. 2008 Dec;7(12):1139-51. 
2. Deloire MSA, Salort E, Bonnet M, et al. Cognitive impairment as marker of diffuse brain abnormalities in early relapsing remitting multiple sclerosis. J Neurol Neurosurg Psychiatry. 2005 Apr;76(4):519-26.

3. Audoin B, Ibarrola D, Ranjeva J-P, et al. Compensatory cortical activation observed by fMRI during a cognitive task at the earliest stage of multiple sclerosis. Hum Brain Mapp. 2003 Oct 1;20(2):51-8.

4. Deloire MSA, Ruet A, Hamel D, et al. MRI predictors of cognitive outcome in early multiple sclerosis. Neurology. 2011 Mar 29;76(13):1161-7.

5. Ruet A, Hamel D, Deloire MSA, et al. Information processing speed impairment and cerebellar dysfunction in relapsing-remitting multiple sclerosis. J Neurol Sci. 2014 Dec 15;347(1-2):246-50.

6. Schmahmann JD. From movement to thought: anatomic substrates of the cerebellar contribution to cognitive processing. Hum Brain Mapp. 1996;4(3):174-98.

7. Allen G, Buxton RB, Wong EC, et al. Attentional Activation of the Cerebellum Independent of Motor Involvement. Science. 1997 Mar 28;275(5308):1940-3.

8. Stoodley CJ, Schmahmann JD. Functional topography in the human cerebellum: a meta-analysis of neuroimaging studies. NeuroImage. 2009 Jan 15;44(2):489-501.

9. Keren-Happuch E, Chen S-HA, Ho M-HR, et al. A Meta-analysis of Cerebellar Contributions to Higher Cognition from PET and fMRI studies. Hum Brain Mapp. 2014 Feb;35(2):593-615.

10. Stoodley CJ, Schmahmann JD. Evidence for topographic organization in the cerebellum of motor control versus cognitive and affective processing. Cortex J Devoted Study Nerv Syst Behav. 2010;46(7):831-44.

11. Buckner RL, Krienen FM, Castellanos A et al. The organization of the human cerebellum estimated by intrinsic functional connectivity. J Neurophysiol. 2011 Nov;106(5):2322-45.

12. Kutzelnigg A, Faber-Rod JC, Bauer J, et al. Widespread demyelination in the cerebellar cortex in multiple sclerosis. Brain Pathol Zurich Switz. 2007 Jan;17(1):38-44.

13. Staff NP, Lucchinetti CF, Keegan BM. Multiple sclerosis with predominant, severe cognitive impairment. Arch Neurol. 2009 Sep;66(9):1139-43.

14. Morgen K, Sammer G, Courtney SM, et al. Evidence for a direct association between cortical atrophy and cognitive impairment in relapsing-remitting MS. NeuroImage. 2006 Apr 15;30(3):891-8.

15. Weier K, Penner IK, Magon S, et al. Cerebellar abnormalities contribute to disability including cognitive impairment in multiple sclerosis. PloS One. 2014;9(1):e86916.

16. Damasceno A, Damasceno BP, Cendes F. The clinical impact of cerebellar grey matter pathology in multiple sclerosis. PloS One. 2014;9(5):e96193.

17. van de Pavert SHP, Muhlert N, Sethi V, et al. DIR-visible grey matter lesions and atrophy in multiple sclerosis: partners in crime? J Neurol Neurosurg Psychiatry. 2016 May;87(5):461-7.

18. Weier K, Till C, Fonov V, et al. Contribution of the cerebellum to cognitive performance in children and adolescents with multiple sclerosis. Mult Scler Houndmills Basingstoke Engl. 2016 Apr;22(5):599-607.

19. Bonnet MC, Allard M, Dilharreguy B, et al. Cognitive compensation failure in multiple sclerosis. Neurology. 2010 Oct 5;75(14):1241-8.

20. Genova HM, Hillary FG, Wylie G, et al. Examination of processing speed deficits in multiple sclerosis using functional magnetic resonance imaging. J Int Neuropsychol Soc JINS. 2009 May;15(3):383-93.

21. Rocca MA, Bonnet MC, Meani A, et al. Differential cerebellar functional interactions during an interference task across multiple sclerosis phenotypes. Radiology. 2012 Dec;265(3):864-73. 
22. Polman $\mathrm{CH}$, Reingold SC, Edan G, et al. Diagnostic criteria for multiple sclerosis: 2005 revisions to the 'McDonald Criteria'. Ann Neurol. 2005 Dec;58(6):840-6.

23. Roxburgh RHSR, Seaman SR, Masterman T, et al. Multiple Sclerosis Severity Score: using disability and disease duration to rate disease severity. Neurology. 2005 Apr 12;64(7):1144-51.

24. Lamargue-Hamel D, Deloire M, Saubusse A, et al. Cognitive evaluation by tasks in a virtual reality environment in multiple sclerosis. J Neurol Sci. 2015 Dec 15;359(1-2):94-9.

25. Smith SM, Zhang Y, Jenkinson M, et al. Accurate, robust, and automated longitudinal and cross-sectional brain change analysis. NeuroImage. 2002 Sep;17(1):479-89.

26. Fischl B, Salat DH, Busa E, et al. Whole brain segmentation: automated labeling of neuroanatomical structures in the human brain. Neuron. 2002 Jan 31;33(3):341-55.

27. Van Leemput K, Maes F, Vandermeulen D, et al. Automated segmentation of multiple sclerosis lesions by model outlier detection. IEEE Trans Med Imaging. 2001 Aug;20(8):677-88.

28. Diedrichsen J. A spatially unbiased atlas template of the human cerebellum. NeuroImage. 2006 Oct $15 ; 33(1): 127-38$.

29. DeLuca J, Chelune GJ, Tulsky DS, et al. Is speed of processing or working memory the primary information processing deficit in multiple sclerosis? J Clin Exp Neuropsychol. 2004 Jun;26(4):550-62.

30. Schmahmann JD, Sherman JC. The cerebellar cognitive affective syndrome. Brain J Neurol. 1998 Apr;121 ( Pt 4):561-79.

31. Manto M, Mariën P. Schmahmann's syndrome - identification of the third cornerstone of clinical ataxiology. Cerebellum Ataxias. 2015;2:2.

32. Cerasa A, Valentino $\mathrm{P}$, Chiriaco $\mathrm{C}$, et al. MR imaging and cognitive correlates of relapsing-remitting multiple sclerosis patients with cerebellar symptoms. J Neurol. 2013 May;260(5):1358-66.

33. Edwards SGM, Gong QY, Liu C, et al. Infratentorial atrophy on magnetic resonance imaging and disability in multiple sclerosis. Brain. 1999 Feb 1;122(2):291-301.

34. Anderson VM, Fisniku LK, Altmann DR, et al. MRI measures show significant cerebellar gray matter volume loss in multiple sclerosis and are associated with cerebellar dysfunction. Mult Scler. 2009 Jan $7 ; 15(7): 811-7$.

35. Calabrese M, Mattisi I, Rinaldi F, et al. Magnetic resonance evidence of cerebellar cortical pathology in multiple sclerosis. J Neurol Neurosurg Psychiatry. 2010 Jan 4;81(4):401-4.

36. Rocca MA, Preziosa P, Mesaros S, et al. Clinically Isolated Syndrome Suggestive of Multiple Sclerosis: Dynamic Patterns of Gray and White Matter Changes-A 2-year MR Imaging Study. Radiology. 2016 Mar;278(3):841-53.

37. Cerasa A, Passamonti L, Valentino P, et al. Cerebellar-parietal dysfunctions in multiple sclerosis patients with cerebellar signs. Exp Neurol. 2012 Oct;237(2):418-26.

38. Chen SHA, Desmond JE. Cerebrocerebellar networks during articulatory rehearsal and verbal working memory tasks. NeuroImage. 2005 Jan 15;24(2):332-8.

39. Bonnet MC, Dilharreguy B, Allard M, et al. Differential cerebellar and cortical involvement according to various attentional load: role of educational level. Hum Brain Mapp. 2009 Apr;30(4):1133-43.

40. Koziol LF, Budding D, Andreasen N, et al. Consensus paper: the cerebellum's role in movement and cognition. Cerebellum Lond Engl. 2014 Feb;13(1):151-77. 


\section{LEGEND OF FIGURE:}

Figure 1: SUIT processing:

a) Cerebellar segmentation and normalisation

b) Grey matter mask resliced into SUIT space 


\section{TABLES}

Table 1: Characteristics of the population

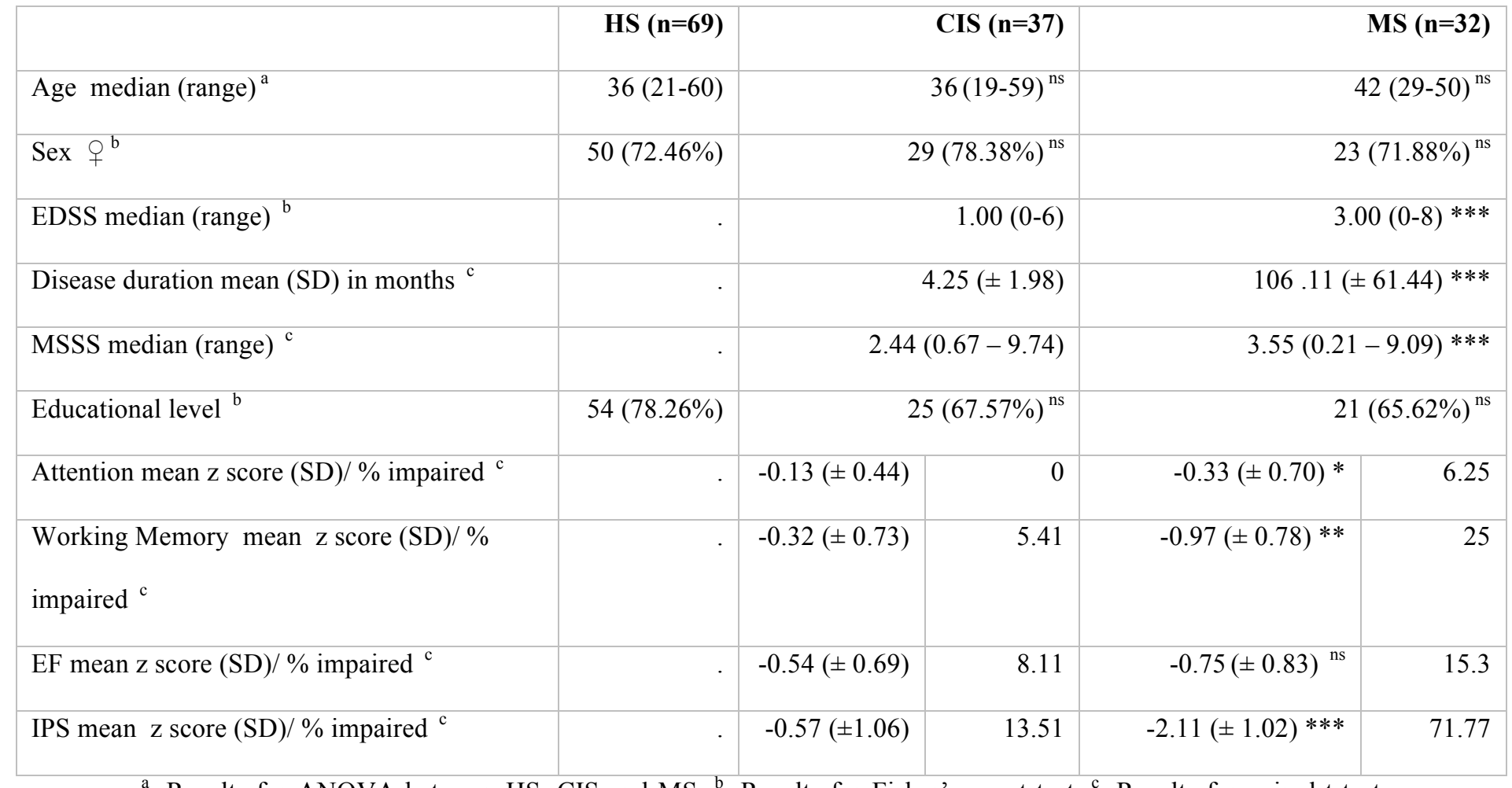

${ }^{a}$ : Results for ANOVA between HS, CIS and MS; ${ }^{b}$ : Results for Fisher's exact test; ${ }^{c}$ : Results for paired t test between persons with clinically isolated syndromes and multiple sclerosis.

Differences between groups: ${ }^{\text {ns }}$ : not significant; $\mathrm{p}>0.05 ; *: \mathrm{p} \leq 0.05 ; * *: \mathrm{p} \leq 0.01 ; * * *: \mathrm{p} \leq 0.001$

EF: executive functions, IPS: information processing speed; SD: standard deviation. 
Table 2: Comparisons of mean normalized volumes $\left(\mathrm{cm}^{3}\right)$ between persons with clinically isolated syndromes (PwCIS), multiple sclerosis (PwMS) and healthy subjects (HS)

\begin{tabular}{|c|c|c|c|c|c|}
\hline & HS $(n=36)$ & PwCIS (n=37) & $\begin{array}{l}\text { PwMS } \\
(n=32)\end{array}$ & $\begin{array}{l}\text { PwMS vs } \\
\text { HS }\end{array}$ & $\begin{array}{l}\text { PwMS vs } \\
\text { PwCIS }\end{array}$ \\
\hline & Mean \pm SD & Mean \pm SD & Mean \pm SD & $\mathrm{p}$ value & $\mathrm{p}$ value \\
\hline Brain $^{a}$ & $\begin{array}{r}1553.37 \pm \\
112.01\end{array}$ & $1550.03 \pm 78.63$ & $\begin{array}{r}1491.88 \pm \\
79.32\end{array}$ & $<0.01$ & $<0.01$ \\
\hline Cerebral GM $^{\text {a }}$ & $\begin{array}{r}823.90 \pm \\
60.76\end{array}$ & $820.03 \pm 57.36$ & $\begin{array}{r}775.82 \pm \\
52.41\end{array}$ & $<0.001$ & $<0.01$ \\
\hline Cerebral WM ${ }^{\mathrm{a}}$ & $\begin{array}{r}729.47 \pm \\
54.61\end{array}$ & $730.00 \pm 35.22$ & $\begin{array}{r}716.06 \pm \\
39.93\end{array}$ & ns & $\mathrm{ns}$ \\
\hline Cerebral T2-WM lesion volume ${ }^{b}$ & . & $8.79 \pm 14.39$ & $\begin{array}{r}22.77 \pm \\
24.77\end{array}$ & N/A & $<0.001$ \\
\hline Cerebellum $^{\mathrm{a}}$ & $\begin{array}{r}180.98 \pm \\
15.08\end{array}$ & $182.54 \pm 15.75$ & $\begin{array}{r}173.41 \pm \\
13.12\end{array}$ & ns & $<0.05$ \\
\hline Cerebellar GM ${ }^{\mathrm{a}}$ & $\begin{array}{r}100.08 \pm \\
12.58\end{array}$ & $99.30 \pm 15.44$ & $\begin{array}{r}91.24 \pm \\
14.66\end{array}$ & $<0.05$ & ns \\
\hline Cerebellar $\mathrm{WM}^{\mathrm{a}}$ & $64.31 \pm 10.14$ & $67.70 \pm 14.05$ & $\begin{array}{r}65.90 \pm \\
11.06\end{array}$ & ns & $\mathrm{ns}$ \\
\hline Infratentorial T2-lesion volume ${ }^{b}$ & . & $0.10 \pm 0.19$ & $0.17 \pm 0.32$ & N/A & $<0.05$ \\
\hline LeftVI GM $^{\text {a }}$ & $9.16 \pm 1.27$ & $9.02 \pm 1.30$ & $8.14 \pm 1.48$ & $<0.01$ & $<0.05$ \\
\hline VermisVI GM $^{\mathrm{a}}$ & $1.50 \pm 0.28$ & $1.55 \pm 0.24$ & $1.36 \pm 0.29$ & ns & $<0.05$ \\
\hline RightVI GM $^{\mathrm{a}}$ & $8.43 \pm 1.08$ & $8.16 \pm 1.03$ & $7.42 \pm 1.21$ & $<0.001$ & $<0.05$ \\
\hline LeftCrusI GM ${ }^{\mathrm{a}}$ & $13.26 \pm 2.25$ & $13.20 \pm 2.70$ & $11.72 \pm 2.77$ & $<0.05$ & $<0.05$ \\
\hline RightVIIb GM ${ }^{\mathrm{a}}$ & $4.28 \pm 1.02$ & $4.16 \pm 1.11$ & $3.72 \pm 0.76$ & $<0.05$ & $\mathrm{~ns}$ \\
\hline
\end{tabular}

ns : not significant; GM : grey matter, WM : white matter; SD: standard deviation.

a: Results for ANOVA between HS, PwCIS and PwMS, with p values for post hoc analyses; ${ }^{\mathrm{b}}$ : Results for $\mathrm{t}$ tests between CIS and MS

No significant differences were found between PwCIS and HS groups. 
Table 3: Correlations and linear regression models between volume metrics and cognitive outcomes

\begin{tabular}{|c|c|c|c|}
\hline Cognitive domain & $\begin{array}{l}\text { MRI parameters included } \\
\text { in models }\end{array}$ & $\mathrm{R}$ univariate analyses & Adjusted $\mathrm{R}^{2}$ model \\
\hline Working memory & Right VIIb & $0.33^{\mathrm{ns}}$ & $0.09^{\circ 0}$ \\
\hline Executive functions & IT T2-lesion volume & $-0.33^{\mathrm{ns}}$ & $0.12^{\circ 0}$ \\
\hline \multirow[t]{7}{*}{ IPS } & Age & $-0.34^{\mathrm{ns}}$ & \multirow[t]{7}{*}{$0.36^{\circ}$} \\
\hline & Cerebral GM & $0.51 * * *$ & \\
\hline & $\begin{array}{l}\text { Cerebral T2-lesion } \\
\text { volume }\end{array}$ & $-0.55 * * *$ & \\
\hline & Cerebellar GM & $0.41 * *$ & \\
\hline & Vermis VI GM & $0.47 * * *$ & \\
\hline & Left Crus II GM & $0.39 * *$ & \\
\hline & Right Crus II GM & $0.40^{* *}$ & \\
\hline
\end{tabular}

Univariate threshold for significance: $*: \mathrm{p} \leq 0.002 ; * *: \mathrm{p} \leq 0.001 ; * * *: \mathrm{p} \leq 0.0001$ after Bonferroni correction;

Multiple linear regression threshold for significance ${ }^{\circ}: \mathrm{p} \leq 0.05 ;{ }^{\circ}: \mathrm{p} \leq 0.01$, significant independent variables are presented in bold type. Linear regression analysis included three hierarchical blocks 1) Clinical data (age and MSSS), 2) Cerebellar volumes (lobule GM volumes and whole infratentorial lesion volume), 3) Cerebral volumes (cerebral WM, cerebral GM and cerebral lesion volume).

MRI: Magnetic Resonance Imaging; IPS: Information Processing Speed, GM: Grey Matter. 